\title{
Integrate the Mitochondrial Genome into the Nuclear Genome Xinkun Wang*
}

Higuchi Biosciences Center, University of Kansas, USA

\begin{abstract}
The mitochondrion is not an ideal location for storing genetic information. Evolution has relocated most of the mitochondrial genome to the nucleus. Transferring all remaining mitochondrial DNA to the nucleus might be an effective approach to cure mitochondrial diseases and slow down aging.
\end{abstract}

Mitochondria are believed to derive from engulfed a-proteobacteria, so it is hardly a surprise that they contain their own genome. What is surprising, though, is that they continue to maintain a tiny fraction of their original genome after 1.3 billion years of symbiosis with their eukaryotic host, when the vast majority of the mitochondrial genome has been either lost completely (no longer needed due to change of lifestyle), or transferred to the host nucleus. While it takes around 3,000 genes to make a mitochondrion in a human cell, the human mitochondrial genome contains only 13 protein-coding genes, among which $5(N D 1, N D 4, N D 5, C Y T B$ and COX1) are universally retained in all organisms (except in organisms for whom aerobic metabolism or part of the electron transport chain is no longer necessary) $[1,2]$. The transfer of mitochondrial DNA (mtDNA) to the nuclear genome is an ongoing process and is still happening today [3]. A survey of human nuclear DNA sequences identified the existence of large mtDNA fragments in many chromosomes [4]. Under certain circumstances that compromise mitochondrial integrity, such as during digestion by lysosomes or when undergoing fusion, pieces of mtDNA can escape from mitochondria and move to the nucleus. Mitochondrial DNA can also be transferred to the nucleus through illicit use of the nucleic acid transport system and through occasional fusion of mitochondrial and nuclear membranes. When ending up in the nucleus, mtDNA can integrate into the host genome through a number of mechanisms, such as double-strand-break repair. When a mitochondrial gene integrates into the nuclear genome at a spot containing intact regulatory elements as well as a mitochondrial targeting sequence, a functional transfer is accomplished.

The horizontal DNA transfer between mitochondria and the nucleus is not a one-way street; it can happen both ways. Just like their proteobacterial ancestors, mitochondria can take up DNA from their environment [5]. The net one-way flow of genetic material from mitochondria to the nucleus and the consequent dramatic reduction of mitochondrial genome over time, however, indicate that evolution favors the migration of genetic information from the cellular "power plant" to the nucleus, the centralized genetic blueprint storage site in a cell. To answer the question of why evolution favors the transfer of genetic information from mitochondria to the nucleus, or more importantly, what is the evolutionary advantage offered by this DNA migration, we have to examine the environment in which mtDNA resides. As the powerhouse of the cell, mitochondria are also the major producer of cellular pollutants, i.e., reactive oxygen species, such as superoxide and hydrogen peroxide. Seated in the midst of these highly reactive molecules, mtDNA is constantly bombarded and therefore has a mutation rate that is much higher than the nuclear DNA. To make the situation worse, the DNA repair system in this organelle is more limited than it is in the nucleus. Clearly, this organelle is not an ideal location for storing DNA molecules.

The detrimental effects of storing key metabolism-related genes in the mitochondria genome become all the more apparent in the process of aging and mitochondrial diseases. The accumulation of mtDNA mutations is a characteristic of the aging process. Although it is still controversial as to whether it is a cause or an effect of aging, it is clear that mitochondrial dysfunction due to mtDNA mutations leads to energy deficits in cells, which increases their vulnerability to the various stressors that ultimately result in cell senescence. Mitochondrial DNA 'mutator' mice, which were genetically altered to elevate the rate of mtDNA mutation, show multiple signs of premature aging $[6,7]$. In the central nervous system, mitochondrial dysfunction due to accumulation of mutant mtDNA in vulnerable brain regions is a salient feature of a number of neurodegenerative diseases, including Alzheimer's disease and Parkinson's disease [8]. In heart and skeletal muscle, accumulated mtDNA mutations lead to energy generation deficiencies and to cardiovascular diseases and myopathies. Besides these well known mitochondrial diseases, inherited or spontaneous mtDNA mutations can lead to a growing list of mitochondrial diseases that lead to visual/hearing difficulties, liver dysfunction, respiratory complications, developmental delay, diabetes, gastro-intestinal disorders, lactic acidosis and vulnerability to infections [2].

If mitochondria are not the ideal location for storing genetic information and accumulation of mtDNA mutations is detrimental to our survival, why do a small number of genes remain in the mitochondria? Several hypotheses have been proposed [9]. The first is the hydrophobicity hypothesis, which postulates that the high hydrophobicity of proteins coded by the remaining mitochondrial genes creates a barrier for their relocation to the nucleus. Some mtDNA coded proteins, such as $\mathrm{NADH}$ dehydrogenase subunit 4 (ND4) of complex I and cytochrome b (CYTB) of complex III, are highly hydrophobic and have multiple transmembrane domains. If their coding genes are transferred to the nucleus, importing them to the mitochondria, while still maintaining functional folding and spatial structure, would not be a trivial task. This hydrophobicity hypothesis can explain why some genes are still retained in the mitochondria. However, not all proteins coded by mtDNA are hydrophobic. The second hypothesis proposes that retaining certain genes inside the mitochondria is for the effective control of their expression and the efficient incorporation

*Corresponding author: Xinkun Wang, Higuchi Biosciences Center, The University of Kansas, 2099 Constant Avenue, Lawrence, KS 66047, USA, Tel: +1 (785) 864-4589; E-mail: xwang@ku.edu

Received March 05, 2012; Accepted March 07, 2012; Published March 09, 2012

Citation: Wang X (2012) Integrate the Mitochondrial Genome into the Nuclear

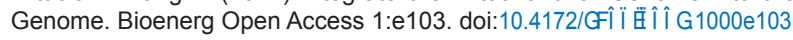

Copyright: (c) 2012 Wang $X$. This is an open-access article distributed under the terms of the Creative Commons Attribution License, which permits unrestricted use, distribution, and reproduction in any medium, provided the original author and source are credited. 
of their protein products into proper protein complexes in the inner membrane. If the same genes are transferred to the nucleus and their mRNA translated in the cytoplasm, the coded polypeptides might be transported into other intracellular membranes (such as endothelial reticulum) and induce local oxidative damage [10,11]. The third proposed mechanism for thwarting mtDNA transfer to the nuclear genome is the use of alternative genetic codons in the mitochondria of some organisms such as animals (including humans).

To counter the use of alternative codons in mtDNA, re-coding mitochondrial genes using the universal genetic code and then transferring them out of the mitochondria and into the nucleus is easily achieved in this day and age. This technique, called allotopic expression, was first employed to transfer the mitochondrial ATP8 gene to the nucleus in yeast cells in 1986 [12]. In human cells this was first accomplished in 2002, when the mitochondrial ATP6 gene, with the addition of a mitochondrial targeting sequence, was recoded and introduced into the nuclear genome [13]. In this endeavor, the recombinant gene was translated in the cytoplasm and the resultant polypeptide was transported back into and processed within mitochondria where it became an integral part of complex V. So far a number of mitochondrial genes, including ATP6 [13-17], ATP8 [12,18], ND1 [19] and ND4 [16,19-23], have been integrated into the nuclear genome. The protein products of these allotopic genes can function within mitochondria. As a result, cellular dysfunction caused by corresponding mutant versions of these genes located in the mitochondrial genome can be complemented. Therefore, allotopic expression could offer an effective strategy for mitochondrial gene therapy to combat mitochondrial diseases caused by mtDNA mutation, such as Leber's Hereditary Optic Neuropathy (LHON), one of the most prevalent mitochondrial diseases. In rodent models of LHON, intraocular injection and in vivo electroporation of re-coded ND4 gene for allotopic expression rescued mitochondrial dysfunction in the affected retinal neurons and prevented impairment of visual function $[21,23]$. Since both in vitro studies and in vivo animal experiments have shown the promise and benefit of using gene transfer from the mitochondria to the nucleus in the treatment of LHON, human clinical trials using this approach of treatment are currently under way [24]. Besides curing mitochondrial diseases such as LHON, moving mtDNA out of the organelle to the nucleus is expected to promote overall health, reduce incidence of diseases (not just mitochondriopathies) and slow down aging [25].

Despite the successes, there are a number of road blocks that need to be overcome if transfer of all 13 remaining human mtDNA structural genes to the nucleus is to occur. First, the high hydrophobicity of some of the mtDNA-coded proteins remains a barrier [26]. Although the import of the allotopically expressed genes into the mitochondria shows that the hydrophobicity barrier can be overcome, most of these genes were not re-coded for reduction of hydrophobicity, so the efficiency of their import is still low. To further overcome this barrier, their hydrophobicity needs be reduced by altering amino acid composition in their transmembrane domain regions. It has been shown that alteration of a couple of key amino acids is sufficient sometimes [27]. To deal with extreme protein hydrophobicity, other approaches, such as the use of inteins [28], are proposed. Second, the import and incorporation of allotopically expressed proteins into the mitochondria are still tricky, because allotopically expressed inner membrane proteins are sometimes mistakenly placed on the outer membrane [29], or they may fail to be incorporated into appropriate mitochondrial protein complexes $[29,30]$. More understanding with regard to the import and assembly of nuclear-genome-encoded mitochondrial proteins is still needed.
In terms of transferring mitochondrial genes to the nucleus, Chlamydomonas reinhardtii, a green alga, is a pioneer, with only 7 metabolically related genes retained in its mitochondrial genome [31]. A number of mitochondrial proteins that are considered too hydrophobic to transfer to the nuclear genome and still coded by the mitochondrial genome in all other species (including humans), have been transferred to the nucleus in $C$. reinhardtii. The smaller number of genes in the C. reinhardtii mitochondrial genome and the allotopic expression of the various mitochondrial proteins described above, make us reach the hypothesis that there is a hierarchical order for the transfer of mitochondrial genes to the nuclear genome and that the most hydrophobic ones are simply the last to be moved out. Without intervention, the transfer of this last batch of genes might take tens of millions of years. With human ingenuity, the transfer may be completed within a much shorter time frame. With a clean mitochondrion and the beauty of one cell - one genome, we may find that many of the health problems we face today would vanish along with the mitochondrial genome. Or, we may find other, previously unappreciated reasons that have prevented the transfer of the last few mitochondrial genes to the nucleus.

\section{Acknowledgment}

The author is supported by NIH grants including NIA P30 AG035982, NIA P01 AG12993, and NICHD P30 HD02528.

\section{References}

1. Adams KL, Palmer JD (2003) Evolution of mitochondrial gene content: gene loss and transfer to the nucleus. Mol Phylogenet Evol 29: 380-395.

2. Naviaux RK: The spectrum of mitochondrial disease. In: Mitochondrial and Metabolic Disorders- A Primary Care Physician's Guide (1st edn.) Oradell: PsyEd Corp.; 1997: 3-10.

3. Timmis JN, Ayliffe MA, Huang CY, Martin W (2004) Endosymbiotic gene transfer: organelle genomes forge eukaryotic chromosomes. Nat Rev Genet 5: $123-135$.

4. Mourier T, Hansen AJ, Willerslev E, Arctander P (2001) The Human Genome Project reveals a continuous transfer of large mitochondrial fragments to the nucleus. Mol Biol Evol 18: 1833-1837.

5. Koulintchenko M, Temperley RJ, Mason PA, Dietrich A, Lightowlers RN (2006) Natural competence of mammalian mitochondria allows the molecula investigation of mitochondrial gene expression. Hum Mol Genet 15: 143-154.

6. Trifunovic A, Wredenberg A, Falkenberg M, Spelbrink JN, Rovio AT, et al (2004) Premature ageing in mice expressing defective mitochondrial DNA polymerase. Nature 429: 417-423.

7. Kujoth GC, Hiona A, Pugh TD, Someya S, Panzer K, et al. (2005) Mitochondria DNA mutations, oxidative stress, and apoptosis in mammalian aging. Science 309: 481-484.

8. Swerdlow RH (2009) The neurodegenerative mitochondriopathies. J Alzheimers Dis 17: 737-751.

9. Daley DO, Whelan J (2005) Why genes persist in organelle genomes. Genome Biol 6: 110.

10. Race HL, Herrmann RG, Martin W (1999) Why have organelles retained genomes? Trends Genet 15: 364-370.

11. Allen JF (2003) The function of genomes in bioenergetic organelles. Philos Trans R Soc Lond B Biol Sci 358: 19-37.

12. Gearing DP, Nagley P (1986) Yeast mitochondrial ATPase subunit 8, normally a mitochondrial gene product, expressed in vitro and imported back into the organelle. EMBO J 5: 3651-3655.

13. Manfredi G, Fu J, Ojaimi J, Sadlock JE, Kwong JQ, et al. (2002) Rescue of a deficiency in ATP synthesis by transfer of MTATP6, a mitochondrial DNAencoded gene, to the nucleus. Nat Genet 30: 394-399.

14. Shidara Y, Yamagata K, Kanamori T, Nakano K, Kwong JQ, et al. (2005) Positive contribution of pathogenic mutations in the mitochondrial genome to the promotion of cancer by prevention from apoptosis. Cancer Res 65: 1655-1663. 
15. Zullo SJ, Parks WT, Chloupkova M, Wei B, Weiner H, et al. (2005) Stable transformation of $\mathrm{CHO}$ Cells and human NARP cybrids confers oligomycin resistance (oli( $(r))$ following transfer of a mitochondrial DNA-encoded oli $(r)$ ATPase6 gene to the nuclear genome: a model system for mtDNA gene therapy. Rejuvenation Res 8: 18-28.

16. Bonnet C, Kaltimbacher V, Ellouze S, Augustin S, Benit P, et al. (2007) Allotopic mRNA localization to the mitochondrial surface rescues respiratory chain defects in fibroblasts harboring mitochondrial DNA mutations affecting complex I or v subunits. Rejuvenation Res 10: 127-144.

17. Ojaimi J, Pan J, Santra S, Snell WJ, Schon EA (2002) An algal nucleus-encoded subunit of mitochondrial ATP synthase rescues a defect in the analogous human mitochondrial-encoded subunit. Mol Biol Cell 13: 3836-3844.

18. Nagley P, Farrell LB, Gearing DP, Nero D, Meltzer S, et al. (1988) Assembly of functional proton-translocating ATPase complex in yeast mitochondria with cytoplasmically synthesized subunit 8 , a polypeptide normally encoded within the organelle. Proc Natl Acad Sci USA 85: 2091-2095.

19. Bonnet C, Augustin S, Ellouze S, Benit P, Bouaita A, et al. (2008) The optimized allotopic expression of ND1 or ND4 genes restores respiratory chain complex activity in fibroblasts harboring mutations in these genes. Biochim Biophys Acta 1783: 1707-1717.

20. Guy J, Qi X, Pallotti F, Schon EA, Manfredi G, et al. (2002) Rescue of a mitochondrial deficiency causing Leber Hereditary Optic Neuropathy. Ann Neurol 52: 534-542.

21. Guy J, Qi X, Koilkonda RD, Arguello T, Chou TH, et al. (2009) Efficiency and safety of AAV-mediated gene delivery of the human ND4 complex I subunit in the mouse visual system. Invest Ophthalmol Vis Sci 50: 4205-4214.

22. Qi X, Sun L, Lewin AS, Hauswirth WW, Guy J (2007) The mutant human ND4 subunit of complex I induces optic neuropathy in the mouse. Invest Ophthalmo Vis Sci 48: 1-10.

23. Ellouze S, Augustin S, Bouaita A, Bonnet C, Simonutti M, et al. (2008) Optimized allotopic expression of the human mitochondrial ND4 prevents blindness in a rat model of mitochondrial dysfunction. Am J Hum Genet 83: 373-387.

24. Lam BL, Feuer WJ, Abukhalil F, Porciatti V, Hauswirth WW, et al. (2010) Leber hereditary optic neuropathy gene therapy clinical trial recruitment: year 1 . Arch Ophthalmol 128: 1129-1135.

25. Aubrey de Grey, Rae M (2007) Ending aging : the rejuvenation breakthroughs that could reverse human aging in our lifetime. 1st edn, St. Martin's Press, New York.

26. Oca-Cossio J, Kenyon L, Hao H, Moraes CT (2003) Limitations of allotopic expression of mitochondrial genes in mammalian cells. Genetics 165: 707-720.

27. Daley DO, Clifton R, Whelan J (2002) Intracellular gene transfer: reduced hydrophobicity facilitates gene transfer for subunit 2 of cytochrome $c$ oxidase. Proc Natl Acad Sci USA 99: 10510-10515.

28. de Grey AD (2000) Mitochondrial gene therapy: an arena for the biomedical use of inteins. Trends Biotechnol 18: 394-399.

29. Perales-Clemente E, Fernandez-Silva P, Acin-Perez R, Perez-Martos A Enriquez JA (2011) Allotopic expression of mitochondrial-encoded genes in mammals: achieved goal, undemonstrated mechanism or impossible task? Nucleic Acids Res 39: 225-234.

30. Bokori-Brown M, Holt IJ (2006) Expression of algal nuclear ATP synthase subunit 6 in human cells results in protein targeting to mitochondria but no assembly into ATP synthase. Rejuvenation Res 9: 455-469.

31. NCBI: Chlamydomonas reinhardtii mitochondrion, complete genome map. [http://tinyurl.com/7f9lttk] 